Conclusion There is value in conducting biological monitoring to assess healthcare workers' exposure to antineoplastic drugs. However, because of the variability in sampling and analytical methods, standardization is recommended to ensure biological monitoring results are comparable.

\section{0-355 THE BIOCHEMICAL EFFECTS OF LEAD CONCENTRATION ON OXIDATIVE STRESS PARAMETERS IN WORKERS}

'Mehrzad Ebrahemzadih, Hamzeh Salehzadeh. 'Neyshabur University of Medical Sciences, Islamic Republic of Iran

\subsection{6/OEM-2021-EPI.9}

Objective The goal of this study was to determine the blood lead concentration in various occupational workers in Sanandaj and its effects on oxidative stress parameters and other blood parameters.

Methods This descriptive-analytical study was carried out in Sanandaj, Iran. A total of 278 subjects were selected as the sample size for this study, of which 64 did not have exposure to occupational lead, and 214 had occupational exposure to lead. The blood lead levels were measured by the atomic absorption spectrophotometer apparatus AA6800. Oxidative stress parameters and other blood parameters were measured by special devices.

Results and Conclusion There was a significant positive relationship between the blood lead concentration and the oxidative stress parameters including lipid peroxidation, malondialdehyde, glutathione, antioxidant serum, and catalase. There was a significant negative relationship between the blood lead concentration and hematological parameters. Also, there was a significant positive relationship between the blood lead level and the lead concentration in the air.

\section{0-399 URINARY PESTICIDE METABOLITE LEVELS AMONG FARM WORKERS IN MALAYSIA: PILOT RESULTS FROM THE IMPRESS STUDY}

'Zulkhairul Naim Bin Sidek Ahmad, Anne-Helen Harding, Hans Kromhout, loannis Basinas, John Cherrie, Karen Galea, Kate Jones, Martie van Tongeren, Roel Vermeulen, Samuel Fuhrimann, Will Mueller, Andrew Povey. ${ }^{1}$ China Medical University, Taiwan

\subsection{6/OEM-2021-EPI.10}

Introduction Pesticides are widely used by farmers in Malaysia but there is limited information on exposures experienced.

Objective To determine Malaysian farmer's exposure from pesticide spray events through the collection and analysis of urine samples.

Method 25 farmers growing either rice $(n=16)$ or vegetables/ fruits $(n=7)$ from the east coast of Malaysia provided two urine samples, one in the morning before they started spraying and the other in the evening of the first day of spraying either with chlorpyrifos $(n=16)$, cypermethrin $(n=18)$ or pyrethroids $(n=21)$. Urinary levels of 3,5,6-Trichloro-2-pyridinol (TCPy), 3-phenoxybenzoic acid (3PBA), and total 2,2, dimethylcyclopropane carboxylic acid (DCVA) were determined by liquid chromatography-mass spectrometry and corrected for urinary creatinine levels.
Results Farmers who used the precursor pesticide had higher levels of urinary metabolites after spraying than those who did not. Urinary TCPy levels of chlorpyrifos applicators were significantly higher post-spraying compared to pre-spraying: median levels and interquartile range (IQR) were 94 (18.6$228.4)$ versus $43(18.8-121.5) \mu \mathrm{mol} / \mathrm{mole}$ creatinine respectively ( $p$ urinary 3PBA levels of pyrethroid users $(n=21)$ were also significantly higher post-spraying than pre-spraying: median levels (IQR) were $1.8(0.6-3.7)$ versus 0.9 (0.5-1.7) $\mu \mathrm{mol} / \mathrm{mole}$ creatinine ( $\mathrm{p}$ Urinary DCVA levels of cypermethrin users $(n=18)$ were significantly higher post-spraying than prespraying : median levels (IQR) were 5.6 (2.3-10.3) versus 1.9 (1.4-4.2) $\mu \mathrm{mol} / \mathrm{mole}$ creatinine (p Pre-spraying urinary TCPy (but not 3PBA or DCVA) levels in farmers who had applied the precursor pesticide were higher than pre-spray levels in farmers who had not $(\mathrm{p}<0.01)$.

Conclusion Current use of pesticides by Malay farmers results in increased exposure that can be detected by urinary metabolite measurement. This suggests further training is needed to reduce exposure in this population.

\section{Breast Cancer}

\section{0-10 BREAST CANCER IN DANISH WOMEN OCCUPATIONALLY EXPOSED TO DIESEL EXHAUST AND POLYCYCLIC AROMATIC HYDROCARBONS}

'Julie Pedersen, Katrine Strandberg-Larsen, Michael Andersson, Johnni Hansen. 'Danish Cancer Society, Denmark

\subsection{6/OEM-2021-EPI.11}

Introduction Both diesel exhaust and specific polycyclic aromatic hydrocarbons (PAHs) have been classified as carcinogenic to humans. However, there is overall limited evidence for an association between these exposures and breast cancer. Objectives To explore the association between occupational exposure to diesel exhaust and PAHs, respectively, and breast cancer, including subtypes.

Methods The study included 38,375 women with incident breast cancer identified in the Danish Cancer Registry, and five breast cancer-free controls per case matched on year of birth who were randomly selected from the Danish Civil Registration System. Full employment history was obtained for all women from a nationwide pension fund, and exposure to diesel exhaust and PAHs was assessed using a job exposure matrix. Conditional logistic regression was used to estimate of odds ratios (ORs) with adjustment for reproductive factors and socioeconomic status.

Results No noteworthy associations were observed for overall breast cancer in women exposed to diesel exhaust. However, diesel exhaust modestly elevated the risk of estrogen receptor negative breast tumours before age 50 years $(\mathrm{OR}=1.26,95 \%$ CI: 1.09-1.46). Duration- and dose-response relationships were also observed for this subtype in this age group. No notable risk patterns were generally observed for $\mathrm{PAH}$ exposure.

Conclusion Occupational exposure to diesel exhaust may elevate the risk of early-onset estrogen receptor negative breast tumours in women. Future studies exploring this association are warranted. 